\title{
Catalysis by Self-Assembled Structures in Emergent Reaction Networks
}

\author{
Gianluca Gazzola ${ }^{1,2}$, Andrew Buchanan ${ }^{1,2}$, \\ Norman Packard ${ }^{1,2}$, Mark Bedau $^{1,2,3 *}$ \\ ${ }^{1}$ ProtoLife Srl, Venezia, Italia \\ ${ }^{2}$ European Center for Living Technology, Venezia, Italia \\ ${ }^{3}$ Reed College, Portland, USA \\ *to whom correspondence should be addressed: mark@protolife.net
}

\begin{abstract}
We study a new variant of the dissipative particle dynamics (DPD) model that includes the possibility of dynamically forming and breaking strong bonds. The emergent reaction kinetics may then interact with self-assembly processes. We observe that self-assembled amphiphilic aggregations such as micelles have a catalytic effect on chemical reaction networks, changing both equilibrium concentrations and reaction frequencies. These simulation results are in accordance with experimental results on the so-called "concentration effect".
\end{abstract}

Keywords: Chemical Reaction Network, Dissipative Particle Dynamics, Selfassembly, Micelle, Concentration Effect.

\section{Introduction}

We seek to understand the properties of networks of chemical reactions that implicitly interact with self-assembled amphiphilic structures ${ }^{1}$. Chemical reaction networks as well as self-assembled amphiphilic structures ${ }^{2}$ are complex systems. Real complex systems in nature often involve the integration of sub-groups of complex systems. The system we study here is one such example. It couples chemical reaction networks with self-assembling amphiphilic structures.

There is ample experimental evidence that such coupled networks exhibit interesting behavior, in particular, that self-assembled amphiphilic structures affect certain chemical reactions. Micelles and other self-assembled structures are known to profoundly increase the rates of certain reactions [11]. The core mechanism is simply that the supramolecular structures increase the local concentration of the reagents, just as

\footnotetext{
${ }^{1}$ An extended version of this article is available at this web page: http://www.ecltech.org/bmcmyp/Data/ECLT/Public/publications.html

${ }^{2}$ Various parameters such as temperature, $\mathrm{pH}$, and critical threshold concentration influence the type (or "phase") of structures that self-assemble from the amphiphiles. In addition to familiar amphiphiles such as fatty acids and phospholipids, other materials self-assemble including biopolymers like oligopeptides [12].
} 
some catalysts do, and thus accelerate reaction rates. For example, hydrophobic reagents will spontaneously concentrate inside micelles, leading to reaction rate acceleration. Sometimes called "micellar catalysis" [24, 27], this catalytic concentration effect has been observed in a variety of chemical systems that involve micelles and reverse micelles [28, 35]. For example, the presence of micelles increases the rate of RNA self-cleavage reactions 100-fold [27]. Many kinds of reactions are catalyzed by micelles, such as redox [21] and hydrolysis reactions [7]. Micellar catalysis is very general and happens with many kinds of self-assembling materials besides amphiphiles. Examples include polymerized and polymeric amphiphiles [24] and dendrimers [7, 23]. Dendrimers are spherical macromolecules that are somewhat similar to micelles, except that while micelles are rather fluid aggregations composed of many amphiphilic molecules held together by the hydrophobic effect, dendrimers are single static structures tightly held together by covalent bonds.

Our goal here is to model and study this kind of catalysis by self-assembled structures in emergent reaction networks, where the dynamics of the network are not explicitly specified in the rules governing the system. Historically, biochemical reaction networks have been modeled using several approaches. Early approaches used networks whose nodes represented chemical species, and lines between nodes represented reactions. Autocatalytic reaction networks also included lines from catalyst nodes to reaction lines, to represent catalyzed reactions $[8,16]$. Other reaction networks have been modeled in immunology: idiotypic networks [9] and more recently, cytokine networks [18]. The chemistry in many of the early network models was abstract. The models intentionally sought to escape from the details of real chemical interactions, for two reasons: capturing the details of real chemical interactions is difficult and immediately begs the question of what level of detail is to be captured, and the results sought from the model were expected (hoped) to be relatively independent from details of the individual chemical reactions; for large networks, the bulk properties of the network (connectivity, scaling, etc.) were hoped to be independent of the details.

More recently, the experimental understanding of reaction networks has been increasing substantially, and there has been an increased awareness of the need to model details of real chemical reactions in order to define and understand biochemical functionality in a given context, e.g., for a cell [29] and for reaction networks with reaction properties based on quantum mechanics [2, 3, 4]. Simultaneously, there has been a growing awareness that chemical reactions cannot by themselves provide a complete picture of biochemical functionality. Structural properties of amphiphilic assemblies must be added to the purely chemical picture. A very rich example is that of lipid structures. These structures are particularly interesting because they have complex phase diagrams, with phase transitions between several different phases, e.g., lipid solution, micelles, and vesicles, because the transitions between these phases may be catalyzed by the presence of other biopolymers, and because some of the phases may themselves have catalytic properties, e.g., for template-directed replication $[19,20,26]$. Finely tuned chemical control of phase transitions in biochemical gels (including more complex gels than simple lipid structures) has been proposed as a general framework for cellular function [25].

In this paper we study a model of interacting microscopic particles that combines relatively simple chemical reaction properties with properties deriving from self- 
assembly processes that can strongly affect the chemical reactions. Interactions between the particles determine both the chemistry and the self-assembly. The macroscopic result of a model simulation is the emergence of a network of chemical reactions that may interact with the self-assembled structures.

\section{The model}

Our model of chemical reaction systems is based on the well-studied dissipative particle dynamics (DPD) framework [13, 14, 22, 30, 31, 32]. In DPD all the elements move in a two- or three-dimensional continuous toroidal space, according to the influences of four pairwise forces, $f_{i}=\sum_{j}\left(F_{i j}^{C}+F_{i j}^{D}+F_{i j}^{B}+F_{i j}^{R}\right)$. These are a conservative "weak" force between pairs of particles, a dissipative force between nearby particles, a spring-like "strong" bond force if two particles are bonded and a random force. Since strong bonds never form or break in the traditional DPD framework, that framework is unable to represent reaction processes that involve forming and breaking strong bonds. We introduced dbDPD, an augmented DPD framework that includes the possibility of making particles react with each other, dynamically forming or breaking strong bonds (see $[1,6]$, to which we refer for a thorough explanation of the model and all its parameters). Relevant parameters occur in the expressions for the weak forces, $F_{i j}^{C}=\alpha_{I J}\left(1-\beta_{I J} r_{i j}\right)$, where $\alpha_{I J}$ and $\beta_{I J}$ are specific to the types of $i$ and $j$ and $r_{i j}$ is the Euclidean distance between the particles, and also include $r_{I J}^{f}$ and $r_{I J}^{b}$, which are respectively the distance beyond and within which the reactive particles of type $I$ and $J$ form and break their strong bond (set to 0.2 and 0.4 in our simulations). Further, we have $l$, the relaxed strong bond length (set to 0.01 ), $\mathrm{k}$, that governs the strength of all strong bonds, represented as Hookean springs $F_{i j}^{B}=k\left(r_{i j}-l\right)$ (set to 100), $\sigma$, a balancing factor between dissipative and random forces (set to 3), and $w^{R}$, a random factor (set to 2.73205) which occur in the expressions $F_{i j}{ }^{D}=\sigma\left(v_{i}-v_{j}\right)\left(1-r_{i j}\right)^{2} / 2$ and $F_{i j}{ }^{R}=o w^{R}\left(1-r_{i j}\right) u$, where $v_{i}$ is the velocity vector of $i$ and $u$ is a uniform random number chosen from the interval $(-1,1)$.

\section{Emergent Chemical Reaction Networks}

Some of the networks in the literature are emergent $[2,16]$, some not. Reaction networks that arise as a result of our chemistry within dbDPD are highly emergent, in the sense that their equilibrium state is very hard to derive without explicit simulation.

Given rules for forming and breaking bonds, together with the constraint of only two or less bonds allowed per particle, we have the basis for a network of linear polymerization reactions. The architecture of the reaction network is determined by the constraints that are set on the process of strong bond formation. The reaction network complexity is controlled with the specification of the pairs of particle types that can 
form strong bonds and of the maximum length $N$ of the polymeric chains resulting from the strong bond formation process.

The simple example we explore is a reaction network identified by the duple $<\{5,4\}, 2>$, namely having two reactive particle types labeled with integers 5 and 4 that can only form chains of length $N=2$, resulting in the architecture shown in Fig. 1.

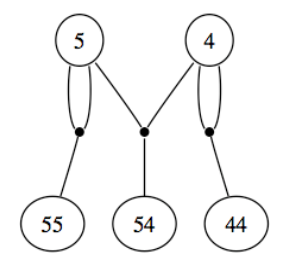

Fig. 1. Architecture of the reaction network for the experiment of polymerization from monomers to dimers.

Each labeled node represents a chemical species that can undergo strong bonding reactions; each solid dot connecting edges from the chemical species represents one of the three possible reactions that can take place: $5+4 \leftrightarrow 54,5+5 \leftrightarrow 55,4+4 \leftrightarrow$ 44. Note that bond forming and bond breaking radii do not influence a chemical reaction network's architecture but play a main role in determining the rate of interaction of reagents in the process of strong bond formation. In our simulations the values of $r_{I J}^{f}$ and $r_{I J}^{b}$, with $r_{I J}^{f}<r_{I J}^{b}$, were the same for all reagents. Thus we can say that all reagents share the same intrinsic reaction rate.

\subsection{Results in a simple network}

The first experiment we report concerns a simple network that contains five particle types: water (type 1), amphiphilic heads (type 2), amphiphilic tails (type 3 ), and two reagents (types 4 and 5). Because it is so simple, this network clearly illustrates the main kinds of interactions between self-assembled structures and emergent reaction networks. The inter-particle interactions are governed by the $\left(\alpha_{I J}, \beta_{I J}\right)$ values specified in Table 1.

Table 1. $\left(\alpha_{I J}, \beta_{I J}\right)$ values for particle interactions in simple network. The particle types 4 and 5 are the monomers that polymerize in the reaction network.

\begin{tabular}{|c|c|c|c|c|c|}
\hline$\left(\alpha_{I J}, \beta_{I J}\right)$ & water & head & tail & 4 & 5 \\
\hline water & $(1,1)$ & & & & \\
\hline head & $(1,1)$ & $(150,1)$ & & & \\
\hline tail & $(4,1)$ & $(15,5)$ & $(5,1)$ & & \\
\hline 4 & $(1,1)$ & $(1,5)$ & $(1,1)$ & $(1,1)$ & \\
\hline 5 & $(1,1)$ & $(1,5)$ & $(1,1)$ & $(1,1)$ & $(1,1)$ \\
\hline
\end{tabular}


Note that "neutral" interactions, e.g., between water and water, are taken to be very weak repulsive interactions, with $\left(\alpha_{I J}, \beta_{I J}\right)=(1,1)$.

The self-assembly process of amphiphilic dimers into micelles can require several time steps in DPD, depending on several factors, such as the temperature of the system and the strength of the weak forces. In our experiments, we wanted to simulate a real chemical system in which lipophilic reagents are placed into an aqueous solution containing micelles that have already formed. In control cases, the amphiphilic dimers were replaced by water and particle initial positions were chosen randomly. All the other DPD parameters were kept the same.

The experiments that we ran were set within a 30x30 toroidal space with 7200 particles, composed of 2/7 reagents, 2/7 amphiphilic dimers and 3/7 water. Simulations were run without allowing the reagents to form bonds, until the amphiphiles aggregated into micelles and the distribution of reagents reached the equilibrium, according to the weak forces that reagents feel towards amphiphiles. Then the particle positions were saved and the simulation was restarted, loading those positions as initial conditions.

One straightforward way to compare the behavior of the system with micelles to a control without micelles is by analyzing the average concentrations of the species identified by the network's nodes at equilibrium. As we can see from Fig. 2 and Table 2 , in the control case concentrations fall into three clearly distinguishable classes. The dominant species are monomers, followed by the only non-palindromic dimer and then by the two palindromic dimers, produced by a self-reaction, which is a reaction between two monomers of the same kind. The experimental case is, in contrast, dominated by the non-palindromic dimer, followed by the two palindromic ones and then by monomers. Note that palindromic dimers are half as concentrated as nonpalindromic dimers in both systems.
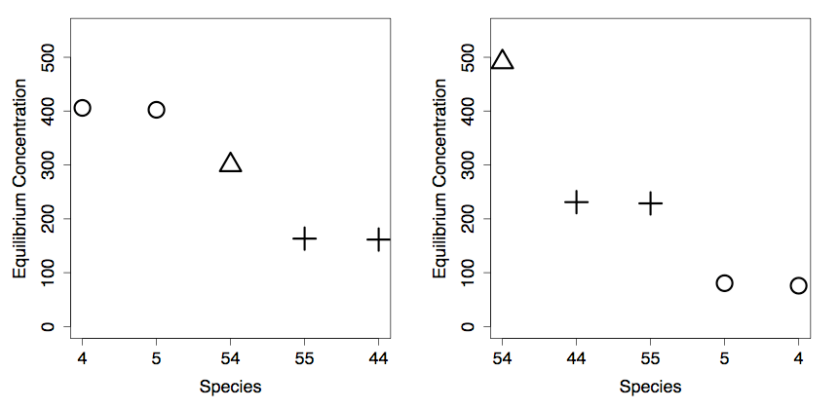

Fig. 2. Equilibrium concentration of different chemical species for a representative dbDPD run of control (left) and experiment (right). The error bars are smaller than the size of the symbols. Note that the triangle has the highest concentration for the experiment, shifted significantly from the control. 
Table 2. Equilibrium concentration for each chemical species as shown in Fig. 2.

\begin{tabular}{|c|c|c|}
\hline Chemical species & Equilibrium concentration & Length \\
\hline \multicolumn{3}{|c|}{ CONTROL } \\
\hline 4,5 & $\sim 404$ & Monomers \\
\hline 54 & $\sim 300$ & Non-palindromic dimer \\
\hline 44,55 & $\sim 162$ & Palindromic dimers \\
\hline 54 & EXPERIMENT & \\
\hline 44,55 & $\sim 491$ & Non-palindromic dimer \\
\hline 5,4 & $\sim 230$ & Palindromic dimers \\
\hline & $\sim 79$ & Monomers \\
\hline
\end{tabular}

Table 3. Reaction frequency for the same run as in Fig. 2 and Table 2.

\begin{tabular}{|c|c|c|}
\hline Chemical reactions & Reaction frequency & Observations \\
\hline \multicolumn{3}{|c|}{ CONTROL } \\
\hline $5+4 \leftrightarrow 54$ & $\sim 103$ & $\begin{array}{c}\text { Monomer + monomer } \leftrightarrow \\
\text { non-palindromic dimer }\end{array}$ \\
\hline $5+5 \leftrightarrow 55$ & $\sim 52$ & $\begin{array}{c}\text { Monomer }+ \text { monomer } \leftrightarrow \\
\text { palindromic dimer }\end{array}$ \\
\hline $4+4 \leftrightarrow 44$ & EXPERIMENT \\
\hline $5+4 \leftrightarrow 54$ & $\sim 88$ & $\begin{array}{c}\text { Monomer + monomer } \leftrightarrow \\
\text { non-palindromic dimer }\end{array}$ \\
\hline $5+5 \leftrightarrow 55$ & $\sim 42$ & $\begin{array}{c}\text { Monomer + monomer } \leftrightarrow \\
\text { palindromic dimer }\end{array}$ \\
\hline $4+4 \leftrightarrow 44$ &
\end{tabular}

We may also compare the reaction dynamics of the two systems, in particular, how frequently reactions happen. Table 3 displays how often each possible reaction happens on average over 50 time steps once the dynamics have reached the equilibrium. In both the control and the experimental case reaction frequencies fall into two classes, one containing the self-reactions and the other one the non-self-reactions. Note that in both cases, the reactions that involve palindromic dimers are half as frequent as those that involve non-palindromic ones. The experimental case shows that the frequency of each reaction is lower than its corresponding one in the control. We now discuss two different observed effects in detail.

Concentration effect: In the control case the only force that can keep the reagents close to each other is due to the (possible) strong covalent bonds formed between two of them. As explained in $[1,6]$, if two reagents come within a distance smaller than their bond forming radius, then they form a strong bond that keeps on existing as long as the reagents' distance is smaller than their bond breaking radius. The reagents are free to float around until they form a strong bond. At this point, the bond strength will determine how long the bonded monomers will stay close to each other enough to keep their bond intact. The weaker the bond, the more likely it will be for it to break in the following time steps, leaving the two resulting monomers free floating again. Apart from the bond strength, nothing affects the survival probability of a dimer. 
In the experimental case, clusters of reagents form because of the weak forces that attract them to micelles. Therefore when the covalent bonds are broken, the monomers don't start to freely diffuse again, but continue to be entrapped in the same cluster, then it is very likely that they are involved in new bonding reactions, possibly with other free monomers in the same cluster.

The probability of existence of a bond depends on several factors. One main factor is the reagents' density (number of reagents over space area). The higher the density, the smaller the average distance between reagents. This probability affects equilibrium concentrations. If bonds are highly likely, then longer polymers are more prevalent. If bonds are unlikely, then monomers are more prevalent. For the reasons we explained above, the reagent density is locally increased by micelles, and that explains why dimers are more concentrated than monomers. By spatially concentrating reagents the micelles act as catalysts. We could also have obtained an analogous result in the control case, by increasing the bond strength.

The concentration effect caused by micelles can be observed from the change in reaction frequencies. Due to the concentration effect, bonded dimers survive for a longer time than when there is no concentration effect, reducing the frequency of the bond breaking reactions. This results in a low number of free reactive monomers, which decreases the frequency of the forward reactions as well.

Palindrome effect. We noticed that the 55 and 44 dimers' concentration is around half of that of the 54 dimer at equilibrium (Fig. 2, Table 2). We also noticed that the frequency of each reaction involving 55's or 44's is half as much of that of the only reaction that involves 54's. The reason is that dimers 54 and 45 have been identified as the same dimer. For all possible pairwise combinations of monomers of type 4 and type 5, if 4's are as many as 5's, there are equal numbers of 44, 45, 54, and 55, so if 45 and 54 are considered identical, their number is double that of 44 and 55 . More generally, one may consider all polymer types of a given length, and see by the same argument that those that are non-palindromic will be doubled by identifying polymers read in one direction with those that are the same when read in the opposite direction.

While the concentration effect concerns the difference between control and experimental equilibria, the palindrome effect concerns the difference between palindromic and non-palindromic $N$-mers in either control or experimental situations. The palindrome effect is actually seen in both the control and the experimental case.

\subsection{Results for a larger network}

We observed qualitatively the same kinds of effects in a wide variety of more complex emergent reaction networks, with higher maximum polymer length and more kinds of reagents.

Cascade effect: The more complex networks illustrated a third effect. Micelles increase the frequency of the reactions involving higher length polymers, as they tend to increase the concentration of their required ingredients compared to the control case. On the way to equilibrium, however, we see that first monomer concentration decreases as dimer concentration rises, then after reaching a maximum, dimer concentration decreases as trimer concentration increases, and so on. This effect moving through successive length polymers we term the "cascade effect". 


\section{Discussion and Conclusion}

We have studied a new variant of dissipative particle dynamics (DPD) with dynamic bond forming and breaking, which we termed "dbDPD". This yields a microscopic mechanism for chemical reactions, from which emerges macroscopic chemical kinetics. The reagents may be represented as nodes on a graph, the reaction network, which also emerges from the microscopic chemical reaction mechanism. The emergent reaction network and reaction kinetics have many of the hallmarks of real reaction networks, e.g., the existence of many side reactions. Here, we have studied a particular class of reactions, polymerization between two monomer types.

DPD is well known as a modeling framework that is suited for studying selfassembled structures from amphiphilic molecules. Our addition of chemical reactions in dbDPD enables the additional study of the interplay between chemical reactions and self-assembly processes.

We report a clear identification of an experimentally known type of micellar catalysis: the concentration effect. Essentially, the effect comes about because lipophilic reagents may aggregate within or near the micelles, effectively increasing their local concentration and changing the equilibrium concentrations of resulting reaction products. In particular, long polymers that have very low equilibrium concentration in the absence of micelles may have very high equilibrium concentration (relative to all other reagents) in the presence of micelles.

In addition to the concentration effect, we identified two other effects that should be experimentally observable: (i) the palindrome effect, the doubling of the concentration of non-palindromic polymers because of the identification of polymers read in one directions with those that are the same when read in the opposite direction, and (ii) the cascade effect, seen when starting with high concentration of monomers: the concentration of monomers goes down as the concentration of dimers increases, then the concentration of dimers reaches a maximum and then decreases as the concentration of trimers increases, and so on.

Future directions for research based on dbDPD include refinement of the microscopic chemical reaction mechanisms to make them more realistic for particular target experiments. We also believe that introduction of variations into reaction products may enable the system to display evolvability. 
Acknowledgements. We thank JohnMcCaskill and Thomas Maeke for an implementation of DPD software from which our software is derived. We benefited from conversations with Harold Fellermann, Ricard Solé, and Martin Hanczyc. Thanks also to the ECLT, which helped facilitate this work and provided an opportunity to present it. This work was supported by the EU in the PACE integrated project, EC-FP6-IST-FET-IP-002035.

\section{References}

1. Bedau M. A., Buchanan A., Gazzola G., Hanczyc M., Maeke T., McCaskill J. S., Poli I. and Packard N. H., Evolutionary design of a DDPD model of ligation. Lecture Notes in Computer Science 3871, (2005) 201-212.

2. Benkö G., Flamm C. and Stadler P.F., A Graph-Based Toy Model of Chemistry. Journal of Chemical Information and Computational Science 43 (2003) 1085-1093.

3. Benkö G., Flamm C. and Stadler P.F., Generic Properties of Chemical Networks: Artificial Chemistry Based on Graph Rewriting, in Banzhaf W., Christaller T., Dittrich P., Kim J. T., and Ziegler J. (Eds.), Advances in Artificial Life: Proceedings of the 7th European Conference on Artificial Life (ECAL), Springer Verlag, Berlin (2003) 10-19.

4. Benkö G., Flamm C. and Stadler P.F., Multi-Phase Artificial Chemistry, in H Schaub, F. Detje, U. Brüggemann (Eds.), The Logic of Artificial Life: Abstracting and Synthesizing the Principles of Living Systems, IOS Press, Akademische Verlagsgesellschaft, Berlin (2004), 16-22.

5. Besold G., Vattulaien I., Karttunen M., Polson J. M., Towards Better Integrators for Dissipative Partycle Dynamics Simulations. Physical Review E 62 (2000) 7611-7614.

6. Buchanan A., Gazzola G., Bedau M. A., Evolutionary Design of a Model of Self-Assembling Chemical Structures, in Natalio Krasnogor, Steve Gustafson, David Pelta and Jose L. Verdegay (Eds.), Elsevier Science, Amsterdam (2007).

7. Delort E., Darbre T. and Reymond J.-L., A Strong Positive Dendritic Effect in a Peptide Dendrimer-Catalyzed Ester Hydrolysis Reaction. Journal of the American Chemical Society 126 (2004) 15642-15643.

8. Farmer J.D., Kauffman S.A. and Packard, N.H.: Autocatalytic Replication of Polymers. Physica D 22 (1986) 50.

9. Farmer, J.D., Packard N.H. and Perelson A.: The Immune System, Adaptation, and Machine Learning, Physica D 22 (1986) 187.

10. Fellerman, H., Rasmussen, S., Ziock, H., Solé, R. (2007). Life cycle of a minimal procell: a dissipative particle (DPD) study. Artificial Life, in press.

11. Fendler J. H. and Fendler E. J., Catalysis in micellar and macromolecular systems. Academic Press, New York (1975).

12. Fung S. Y., Keyes C., Duhamel J. and Chen P.: Concentration Effect on the Aggregation of a Self-Assembling Oligopeptide. Biophysical Journal 85 (2003) 537-548

13. Groot R. and Warren P., Dissipative particle dynamics: bridging the gap between atomistic and mesoscopic simulations. Journal of Chemical Physics 107 (1997) 4423-4435.

14. Hoogerbrugge P. and Koelman J.: Simulating microscopic hydrodynamic phenomena with dissipative particle dynamics. Europhysics Letters 19 (1992) 155-160.

15. Jury S., Bladon P., Cates M., Krishna S., Hagen M., Ruddock N. and Warren P.: Simulation of amphiphilic mesophases using dissipative particle dynamics. Physical Chemistry and Chemical Physics 1 (1999) 2051-2056.

16. Kauffman S. A., Autocatalytic sets of proteins. Journal of Theoretical Biology, 119 (1986) $1-24$. 
17. Kranenburg M., Venturoli M. and Smit B., Phase behavior and induced interdigitation in bilayers studied with dissipative particle dynamics. Journal of Physical Chemistry 107 (2003) 11491-11501.

18. Kuby J.: Immunology, 3rd ed.,W. H. Freeman, New York (1997).

19. Luisi P.L., Giomini M., Pileni M., and Robinson B., Reverse micelles as hosts for proteins and small molecules. Biochimica and Biophysica Acta 947 (1988) 209-246.

20. Luisi P.L., Walde P. and Oberholzer T., Enzymatic synthesis in self-reproducing vesicles: An approach to the construction of a minimal cell. Berichte der Bunsengesellschaft für Physikalische Chemie 98 (1994) 1160-1165.

21. Mallick K., Jewrajka S., Pradhan N. and Pal T., Micelle-catalysed redox reaction. Current Science 80 (2001) 1408-1412

22. Marsh, C.: Theoretical aspects of dissipative particle dynamics. University of Oxford, Ph.D. Thesis (1998).

23. Martin K. I. and Twyman L. J., Acceleration of an aminolysis reaction using a PAMAM dendrimer with 64 terminal amine groups. Tetrahedron Letters 42 (2001) 1123-1126.

24. Oehme G., Grassert I., Paetzold E., Fuhrmann H., Dwars T., Schmidt U. and Iovel I., The Effect of Assembled Amphiphiles on Catalytic Reactions in Aqueous Media. Kinetics and Catalysis, 44 (2003) 766-777.

25. Pollack G. H., Cells, Gels and the Engines of Life: A New, Unifying Approach to Cell Function, Ebner \& Sons, Seattle, WA, USA (2001).

26. Rasmussen S., Chen L., Stadler B. and Stadler P., Proto-organism kinetics: Evolutionary dynamics of lipid aggregates with genes and metabolism, Origins of life and evolution of the biosphere (in press).

27. Riepe A., Beier H. and Gross H. J., Enhancement of RNA self-cleavage by micellar catalysis. FEBS Letters 457 (1999) 193-199.

28. Ruasse M.-F., Blagoevab I. B., Garcia-Rio R. C. L. G., Leis J. R., Marques A., Mejuto J. and Monnier E., Organic reactions in micro-organized media: Why and how? Pure and Applied Chemistry, 69 (1997) 1923-1932.

29. Shilling C. H. and Palsson B. O., The underlying pathway structure of biochemical reaction networks, Proceedings of the National Academy of Science 95 (1998) 4193-4198.

30. Shillcock J. and Lipowsky R., Equilibrium structure and lateral stress distribution from dissipative particle dynamics simulations. Journal of Chemical Physics 117 (2002) 5048-5061.

31. Trofimov S., Nies E. and Michels M., Thermodynamic consistency in dissipative particle dynamics simulations of strongly nonideal liquids and liquid mixtures. Journal of Chemical Physics 117 (2002) 9383-9394.

32. Vattulainen I., Karttunen M., Besold G. and Polson J.: Integration schemes for dissipative particle dynamics simulations: From softly interacting systems towards hybrid models. Journal of Chemical Physics 116 (2002) 3967-3979.

33. Yamamoto S., Maruyama Y. and Hyodo S.: Dissipative particle dynamics study of spontaneous vesicle formation of amphiphilic molecules. Journal of Chemical Physics 116 (2002) $5842-5849$.

34. Yamamoto S., Hyodo, S.: Budding and fission dynamics of two-component vesicles. Journal of Chemical Physics 118 (2003) 7937-7943.

35. Zingaretti L., Boscatto L., Chiacchiera S. M. and Silber J. J. : Kinetics and mechanism for the reaction of 1-chloro-2,4-dinitrobenzene with n-butylamine and piperidine in AOT/nhexane/water reverse micelles. Arkivoc X (2003) 189-200. 